\title{
Reconnaissance de représentativité et quête de reconnaissance
}

Le cas de l'Organisation des transporteurs routiers européens

Recognition of representativeness and quest for representativeness. The case of the European Road Transport Organisation (OTRE)

\section{Stéphane Carré}

\section{(2) OpenEdition}

\section{Journals}

Édition électronique

URL : http://journals.openedition.org/travailemploi/5754

DOI : 10.4000/travailemploi.5754

ISSN : 1775-416X

Éditeur

DARES - Ministère du Travail

Édition imprimée

Date de publication : 15 septembre 2012

Pagination : 65-74

ISSN : 0224-4365

\section{Référence électronique}

Stéphane Carré, «Reconnaissance de représentativité et quête de reconnaissance », Travail et Emploi [En ligne], 131 | 2012, mis en ligne le 01 décembre 2014, consulté le 20 avril 2019. URL : http:// journals.openedition.org/travailemploi/5754; DOI : 10.4000/travailemploi.5754 


\title{
Reconnaissance de représentativité et quête de reconnaissance. Le cas de l'Organisation des transporteurs routiers européens
}

\author{
Stéphane Carré(*)
}

Une préoccupation essentielle de l'OTRE, organisation patronale du secteur routier créée en 2000 à la suite d'un conflit interne à la Fédération nationale des transports routiers (FNTR), a été durant dix ans sa reconnaissance officielle de représentativité, reconnaissance lui permettant de participer aux négociations collectives en commission paritaire de branche. Dans le même temps, l'organisation s'est concentrée, d'un point de vue revendicatif, sur la politique routière de l'État français, axe a priori sans lien étroit avec une possible reconnaissance de représentativité. Mais il apparaît que l'obtention de cette reconnaissance par l'État autorise en fait l'organisation patronale à participer pleinement aux consultations institutionnelles ou informelles que l'Administration initie en matière routière.

L'OTRE est un syndicat professionnel de transporteurs (voir encadré 1). En 2000, alors qu'un conflit ouvert éclatait entre certains transporteurs routiers de marchandises et les pouvoirs publics, aboutissant à la mise en place de barrages routiers, le refus d'une partie de la base, pour l'essentiel des petits transporteurs, de lever les barrages à la demande de l'organisation professionnelle à l'initiative de cette action, la Fédération nationale des transports routiers, aboutissait à l'exclusion des syndicats locaux de transporteurs tenus pour responsables de cette fronde. Il fut alors décidé de la constitution d'une nouvelle fédération regroupant les syndicats exclus, qui allait demander à l'État une reconnaissance de représentativité(1). Il faudra dix ans d'un dialogue tumultueux avec les pouvoirs publics pour obtenir cette reconnaissance administrative. Ce dialogue a fait l'objet d'une recherche, qui est le matériau principal de cet article (voir encadré 2).

(*) IUT de St-Nazaire, UMR CNRS 6297 «Droit et changement social»; Stephane.Carre@univ-nantes.fr

(1) Début septembre 2000, la FNTR était engagée dans une épreuve de force avec l'État en matière de fiscalité routière, sur le remboursement partiel de la Taxe intérieure sur les produits pétroliers. Un accord avec l'État ayant été trouvé, il fut publiquement annoncé par l'organisation patronale sans que «la base» soit consultée, si bien que celle-ci contesta le comportement du délégué général de la FNTR. L'organisation décida très rapidement l'exclusion d'un certain nombre de syndicats locaux. Dans les semaines suivantes, à défaut d'accepter un retour vers la FNTR, les membres des syndicats exclus se réunirent à Toulouse et décidèrent de ne pas rallier une organisation nationale existante mais de créer un nouveau syndicat.

\section{Encadré 1 \\ L'organisation de I'OTRE}

L'Organisation des transporteurs routiers européens est un syndicat patronal, c'est-à-dire une organisation syndicale au sens du Code du travail (L. 2111-1 et s. cod. trav.). Comme nous l'avons déjà indiqué, elle est créée en octobre 2000 , à la suite de l'exclusion de syndicats locaux affiliés à la Fédération nationale des transporteurs routiers (FNTR) et regroupe au départ essentiellement des syndicats du sud-ouest de la France et, dans une moindre mesure, du sud-est. Les adhérents de l'OTRE sont précisément des syndicats départementaux, lointains héritiers des Groupements professionnels routiers (GPR, voir par ailleurs encadré 3 plus bas dans le texte). Quant à leurs adhérents, il s'agit principalement de petites et moyennes entreprises (PME) du transport routier de marchandises, mais aussi d'autocaristes. Par la suite, l'organisation va s'étendre aux autres régions françaises, sur la base de structures régionales. Ses statuts permettent également l'adhésion directe d'un transporteur à la structure nationale, située à Bordeaux, dès lors qu'il n'existe pas encore de syndicats départementaux ou régionaux. L'OTRE revendiquait 3221 entreprises adhérentes, directement ou non, en 2008; les services de l'administration du Travail n'en retiennent que 1886 la même année (source : bureau des relations collectives du travail).

Par ailleurs, les statuts de l'organisation, portant en cela la marque des conditions de sa naissance, rendent particulièrement protecteurs des intérêts des structures locales toute tentative d'exclusion d'un syndicat affilié à l'initiative de la structure nationale : délais de réflexion, décision collégiale, possibilité d'appel, etc. 
Le positionnement de l'OTRE sur la défense des intérêts professionnels des PME du transport routier la place en concurrence avec l'Union nationale des organisations syndicales des transporteurs routiers automobiles (Unostra). L'OTRE et l'Unostra, ainsi que l'union TLF (Entreprises de transport et de logistique de France) vont cependant s'allier au travers d'une structure commune, l'Union des professionnels de la route (UPR), en 2004, et mener des actions conjointes, contrant ainsi une autre union d'organisations professionnelles dominée par la FNTR, I'Union des fédérations de transport (UFT). En 2007, plusieurs unions régionales Unostra se rapprochent ostensiblement de l'OTRE. En 2008, la rupture est consommée entre ces unions régionales et la structure nationale Unostra. Des syndicats locaux Unostra, situés au nord de la France (Picardie, Nord-Pas-de-Calais), demandent alors leur rattachement à l'OTRE.

L'Union TLF, alliée de longue date de l'OTRE, l'a clairement soutenue dans sa quête de reconnaissance, notamment lors des enquêtes administratives de représentativité et en commission mixte paritaire de branche. À l'inverse, l'OTRE et la FNTR ont dénié leur représentativité respective tout au long des années 2000.

\section{Encadré 2 \\ Méthodologie de l'étude}

La présente étude est issue d'une recherche de terrain analysant le discours de l'Organisation des transporteurs routiers européens concernant son éventuelle représentativité, entre 2000 et 2009. Une synthèse en est présentée dans le cadre du rapport La construction de la représentativité patronale remis à la Dares (Direction de l'animation de la recherche, des études et des statistiques) et réalisé sous la direction de Nicole MAGGI-Germain et JeanPierre Le CRom (2011); le compte-rendu exhaustif de la recherche est placé en annexe du rapport (pp. 285-345).

L'enquête a consisté en :

- l'analyse exhaustive sur dix ans des publications de l'organisation dès leur création : en particulier son mensuel, sa lettre hebdomadaire, le récolement de l'ensemble des discours de conclusion des congrès annuels, l'analyse du site web de l'organisation;

- le dépouillement des archives de l'OTRE, au siège de l'organisation à Bordeaux (courriers entrants, sortants, photocopies de mails, etc.) de façon systématique de 2000 à 2006 et, de façon plus ciblée, sur la seule demande de reconnaissance en représentativité de 2007 à 2009;

- des entretiens semi-directifs avec la secrétaire nationale de l'organisation, la responsable administrative, le responsable de la communication et le président de la commission mixte paritaire du secteur des transports routiers.
Pour une entreprise de représentation, comme le sont les organisations professionnelles, la question de la représentativité est cruciale : il s'agit pour elles d'être reconnues comme étant à l'image du groupe qu'elles représentent. Leur rôle, établi par le droit, est celui d'un syndicat, c'est-à-dire «l'étude et la défense des droits ainsi que des intérêts matériels et moraux, tant collectifs qu'individuels, des personnes mentionnées dans leurs statuts » (art. L. 2131-1 cod. trav.). Ce sont les intérêts de toute une profession qui seront ainsi en principe défendus par l'organisation (loi du 12 mars 1920). Cependant, en pratique, comment s'opère, via l'organisation, la rencontre de ces intérêts professionnels avec le restant de la société civile et les instances politiques?

A priori, la reconnaissance de l'organisation par ses interlocuteurs - autrement dit la possibilité toute factuelle d'être entendue, sinon écoutée - sousentend qu'elle est considérée, par ses partenaires, comme représentative du groupe professionnel au nom duquel elle s'exprime. En principe, cette représentativité factuelle (par opposition à la représentativité juridique) de l'organisation, en amont du syndicat, concentre donc toute la légitimité de l'organisation professionnelle à représenter, en aval, la profession auprès d'autres instances. C'est parce qu'une organisation ad hoc - avant même d'être constituée en syndicat - est reconnue représentative d'une profession par ses interlocuteurs potentiels qu'elle devient légitime pour représenter, en aval - c'est-à-dire une fois le syndicat créé - la profession auprès d'autres instances. Cependant, cette représentativité factuelle de l'organisation n'aboutit pas forcément à sa reconnaissance en droit, $i$. e. à sa représentativité juridique. Et à l'inverse, la reconnaissance juridique dont le syndicat peut faire l'objet ne signifie pas que l'organisation soit toujours considérée comme représentative dans les faits par les acteurs en présence. Il s'agit de deux questions distinctes.

La représentativité revendiquée par une organisation professionnelle participe d'une «identité pour soi-même» alors que la représentativité en droit participe d'une «identité pour autrui», d'un processus d'étiquetage aboutissant à une reconnaissance socialement établie (Dubar, 2010, p. 103 et s.). Ainsi, le droit du travail français indique que certaines organisations syndicales sont «représentatives» et attache à cette représentativité des effets de droit (art. L. 2122-1 
et $\mathrm{s}$. cod. trav.) ${ }^{(2)}$. La reconnaissance juridique de la représentativité accorde de la sorte à l'OTRE un ensemble de droits, principalement en matière de dialogue social. Cependant, la reconnaissance sociale dont fait ainsi l'objet l'organisation, en particulier sa capacité à se faire entendre, ne doit pas être assimilée aux seuls effets de droit induits de la reconnaissance juridique de représentativité. Quoique l'action revendicative de l'organisation se soit concentrée sur la politique routière de l'État français et non sur le dialogue social, il s'avère que le syndicat n'a pourtant eu de cesse d'obtenir cette reconnaissance de représentativité, en en faisant un axe essentiel de son action. Ce décalage peut s'expliquer par la fonction réelle accordée par la pratique administrative à la reconnaissance de représentativité. Elle garantit une reconnaissance sociale beaucoup plus large de l'organisation auprès de ses interlocuteurs.

La pratique juridique et administrative autour d'une législation, ici les règles en matière de reconnaissance de représentativité, peut donner une dimension très différente à la loi, faisant de celle-ci un enjeu important pour la reconnaissance sociale d'une organisation professionnelle( ${ }^{(3)}$. La représentativité juridique devient alors le symbole d'une reconnaissance sociale que l'organisation, se disant victime d'une injustice, pouvait considérer comme un élément constitutif de son identité (4).

(2) Une représentativité de fait ne doit pas être confondue avec la représentativité en droit, aux conditions fixées par le Code du travail. Cette dernière notion s'appuie sur un certain nombre de critères légaux (L. 2121-1 cod. trav.) que la jurisprudence et l'Administration ont interprétés de telle sorte que le nombre d'adhérents, notamment par rapport aux effectifs de la profession, en est devenu le critère largement dominant (Flament, 2009; HéAs, 2011). Cette approche n'est pas celle des sociologues qui, sans méconnaître l'importance d'une implantation syndicale, s'interrogent aussi sur la qualité de la représentation exercée par l'organisation, au nom des adhérents, pour discuter de la réelle représentativité de l'organisation à l'aune de leurs aspirations, sinon de celles de l'ensemble du groupe professionnel représenté (BUNEL, 1996, p. 3 ; COURTY, 2006, p. 44 et s.; OfFERLÉ, 2009, p. 46 et s.).

(3) Souvent, la sociologie juridique se focalise sur les phénomènes d'application partielle du droit. Mais l'écart à la règle peut à l'inverse consister en une influence élargie : la règle opère au-delà de ce que laisse à penser l'écrit qui la pose ou les juges qui l'interprètent.

(4) Nous entendons ici l'identité sociale comme un processus de construction sociale et symbolique permettant de marquer l'unité du groupe et de se différencier d'autres groupes (SURDEZ, Voegti, Voutat, 2010, p. 19 et s. Sur la notion controversée d'identité en sociologie, et plus spécialement sur les processus de reconnaissance d'une identité collective, notamment des identités professionnelles, voir aussi : BERGER, LUCKMANN, 2006; LAPIERRE, 1984, p. 195 ; DUBAR, 2010).

\section{Les conséquences juridiques d'une reconnaissance de représentativité dans le transport routier de marchandises (TRM) : des effets circonscrits}

À parcourir le Code du travail, la représentativité des syndicats est une question centrale du droit syndical depuis qu'il n'existe plus de présomption irréfragable de représentativité au bénéfice de certaines organisations ouvrières. Mais qu'en est-il pour une structure patronale telle que l'OTRE, tant au niveau national qu'au niveau de ses structures locales?

D'un point de vue juridique, l'obtention d'une représentativité au niveau national présente des apports limités. De façon certaine, ils sont circonscrits à trois institutions. Il s'agit principalement de la possibilité de participer, dans le cadre de la commission mixte paritaire des transports routiers, à la négociation d'accords de branche susceptibles de faire l'objet d'une extension (art. L. 2261-19 cod. trav.). À cela s'ajoute la possibilité d'être membre du Comité national routier( ${ }^{(5)}$ et de la Commission consultative nationale pour la délivrance des attestations de capacité professionnelle ${ }^{(6)}$. Bien entendu, la possibilité de participer aux négociations en commission mixte paritaire n'est pas un enjeu négligeable. Elle n'est cependant qu'un aspect extrêmement étroit de la reconnaissance d'une organisation professionnelle auprès de ses interlocuteurs. En effet, au niveau local, il lui est loisible d'entretenir des rapports informels avec les mairies, les conseils généraux et régionaux ou les représentants de l'État, qu'il s'agisse des services préfectoraux ou des services déconcentrés des ministères, pour régler des problèmes locaux. Mais il apparaît également que la participation à certaines instances officielles du secteur des transports

(5) Décret n 89-169 du 13 mars 1989, modifié par le décret n $2001-929$; arrêté du 16 octobre 2008 ( $J O$ du 4 novembre). (6) Arrêté du 15 novembre 1999 ( $J O$ du 30 novembre). Au niveau régional, les Commissions consultatives régionales pour la délivrance des attestations de capacité professionnelle réunissent notamment des représentants des organisations professionnelles «les plus représentatives au niveau national». 
n'exige en rien une telle représentativité nationale ou même locale ${ }^{(7)}$.

Par-delà ces commissions ou comités de droit public, dont l'organisation est fixée par la loi, existent des institutions de droit privé. Et là encore, l'exigence juridique d'une représentativité n'est pas toujours explicitement prise en compte. Précisément, ces organismes, souvent constitués sous la forme d'une association loi 1901, vont assez librement fixer dans leurs statuts quels sont les membres constitutifs de l'association, ainsi que le mode d'adhésion ultérieur à l'organisme, malgré quelques exigences légales en matière de représentativité( ${ }^{(8)}$.

Enfin, en ce qui concerne la participation, côté patronal, à la gestion d'organismes de prévoyance (Carcept) ou de collecte des fonds de la formation professionnelle (Fongecfa, etc.), la situation apparaitt assez incertaine. Certes, il peut exister une exigence légale de représentativité (art. L. 2261-4 cod. trav.). Mais les textes conventionnels qui précisent en pratique la gestion de ces institutions paraissent laisser aux organisations patronales signataires le contrôle de l'institution, paritairement avec les organisations salariales, et avec l'aval éventuel des pouvoirs publics. Aussi, l'effective participation à la gestion de ces organismes repose surtout sur la cooptation ou des compromis internes ${ }^{(9)}$.

(7) Ainsi pour les Commissions régionales de sanctions administratives, le décret $n^{\circ} 84-139$ du 24 février 1984 relatif au Conseil national des transports, aux Comités régionaux des transports et aux Commissions régionales des sanctions administratives; ainsi encore pour la Commission nationale des sanctions administratives établie au sein du Conseil national des transports. Il apparaît aussi que la participation au Comité des usagers du réseau routier national n'exige pas que les organisations professionnelles fassent la démonstration de leur représentativité (décret $n^{\circ}$ 2009-1102). Il en est de même des commissions consultatives chargées, dans chaque département, de veiller à l'application des dispositions sur la sécurité de la conduite et sur le temps de travail, et des Commissions départementales de sécurité routière (R. 411-11 Code de la route).

(8) Les Observatoires régionaux des transports sont ainsi en général constitués en associations loi 1901. Leurs statuts prévoient en pratique quels sont les membres originels ainsi que les modalités d'adhésion ultérieure. La même remarque s'applique aux organismes de formation tels que Promotrans ou l'AFT-IFTIM.

(9) Par exemple, pour la Carcept-Prévoyance, nous sommes en face d'une personne morale de droit privé administrée paritairement par les membres adhérents ou participants (L. 922-1 Code de la sécurité sociale). Mais la Carcept doit répondre aux conditions particulières de fonctionnement posées par le décret $n^{\circ} 55-1297$. Ce dernier dispose que les administrateurs sont nommés par le ministre des Transports sur proposition des organisations syndicales les plus représentatives. Cependant la Carcept fonctionne en vertu d'un accord entre partenaires sociaux. Le dernier en date (17 décembre 2001) stipule que le conseil d'administration est composé de quinze membres; les membres employeurs sont nommés par le ministre, mais désignés par le Medef, la Confédération générale du patronat des petites et moyennes entreprises (CGPME) et l'Union professionnelle artisanale (UPA) sur proposition des organisations professionnelles «compétentes», autrement dit les organisations signataires de l'accord...
Malgré les effets apparemment limités d'une reconnaissance administrative de représentativité au niveau national, on pourrait penser que l'obtention du label est stratégique pour l'organisation, compte tenu d'une possible focalisation de son action sur la négociation sociale. Nous allons pourtant voir qu'il n'en a rien été entre 2000 et 2009.

\section{L'action revendicative de l'OTRE : des interventions en faible synergie avec une reconnaissance de représentativité}

L'organisation déploie une action très diversifiée qui ne nécessite aucunement, d'un point de vue juridique, une reconnaissance de représentativité nationale. D'abord, l'organisation développe des modes d'action qui ne sont pas spécifiquement centrés sur les institutions pour lesquelles il est nécessaire d'avoir une reconnaissance administrative de représentativité. L'activité quotidienne de l'organisation, indépendamment des relations avec les adhérents, prend la forme de rapports plus ou moins informels avec certains élus nationaux (députés, sénateurs) dans le cadre d'actions de lobbying, d'alliances conclues avec d'autres organisations professionnelles (Entreprises de transport et de logistique de France [TLF], Union nationale des organisations syndicales des transporteurs routiers automobiles [Unostra], Association française du transport routier international [AFTRI], etc.) et de relations ponctuelles entretenues avec les fonctionnaires de différents ministères, en particulier ceux du ministère des Transports et, au niveau local, les Préfectures, les Directions régionales de l'équipement ou les services des Conseils généraux ou régionaux. Ces rapports informels, qu'attestent en permanence des échanges épistolaires ou des rencontres, ont souvent pour motif des questions pratiques (itinéraires de détournement, barrières de dégel, etc., voir encadré 3). Ils illustrent la reconnaissance informelle dont l'organisation bénéficie. 


\section{Encadré 3}

\section{Les organisations professionnelles et la régulation du secteur routier}

La Fédération nationale des transports routiers (FNTR), I'Unostra, l'OTRE et Transport et Logistique de France forment aujourd'hui les principales organisations professionnelles du transport routier de marchandises. II faut ajouter la Fédération nationale des transports de voyageurs (FNTV), pour les autocaristes et l'Union des transports publics, pour les transports urbains et ferroviaires. La FNTR est l'organisation dominante et historique du secteur. Cette organisation apparaît en 1945. En 1947 est également constituée l'Association française du transport routier international (AFTRI), association d'organisations professionnelles routières, qui les représentent auprès de l'International Road Union, à Genève.

Pour comprendre comment la profession s'est organisée et structurée en France, un élément crucial de compréhension est de se souvenir que l'activité du transport routier a longtemps fait l'objet d'un encadrement serré de la part des pouvoirs publics, dès l'instant qu'il est apparu nécessaire de coordonner les différents moyens de transport, notamment en vue de limiter le développement anarchique du mode routier, notamment face aux chemins de fer (décret-loi du 19 avril 1934). L'État va chercher à obtenir des organisations professionnelles qu'elles participent activement à l'encadrement de la profession (néo-corporatisme). Ainsi, après-guerre sont constitués des Comités techniques départementaux des transports, où, à côté de fonctionnaires et d'élus, les transporteurs sont représentés. Ces comités tiennent le registre des transports, délivrent les autorisations de transports et contrôlent la bonne application de tarifs réglementaires. Dès 1949 est également constitué le Comité national routier, qui va perdurer jusqu'à aujourd'hui. En 1956, les Groupements professionnels routiers, apparus en 1949, prennent leur physionomie définitive. Ils absorbent en particulier les syndicats locaux de transporteurs, délivrent les carnets de feuilles de route et contrôlent finalement la tarification routière (dont la Tarification routière obligatoire, apparue en 1961). À noter que c'est en 1956 qu'est constituée l'Unostra, par le regroupement de trois organisations professionnelles. Elle se veut le défenseur des petits transporteurs. Beaucoup de ces adhérents viennent en fait de la FNTR.

À partir des années soixante-dix, et surtout des années quatre-vingt, a lieu un mouvement de libéralisation des transports routiers. Progressivement le contingentement des opérations de transport routier disparaît. La disparition de ce contingentement et de la Tarification routière obligatoire (TRO) est consommée en 1986. Les Groupements professionnels routiers (GPR) et les Comités techniques départementaux des transports (CTDT) disparaissent également. Les organisations professionnelles, notamment la FNTR, perdent le poids qu'elles avaient pu obtenir par la cogestion avec les pouvoirs publics de la régulation du secteur. C'est à la fin de cette période de transition qu'est constitué Transport et Logistique de France (1998).

En outre, l'action revendicative de l'OTRE ne concerne pas spécialement ce pourquoi la reconnaissance de représentativité a été instituée, c'est-à-dire la mise en œuvre d'un dialogue entre représentants patronaux et salariaux, aboutissant à des conventions collectives et des accords susceptibles d'une extension. Et ce point est essentiel : le champ social n'a pas été au cœur des revendications de l'organisation. Certes, à prendre pour étalon les sujets d'information développés dans le mensuel de l'organisation, entre janvier 2001 et septembre 2009, les rubriques à caractère social occupent une importance certaine. Elles représentent 271 occurrences pour 288 items concernant d'autres thèmes, soit $48 \%$ du total (559 occurrences). Cependant, cette importance des sujets sociaux dans le mensuel relève des services que rend l'organisation à ses adhérents et, plus globalement, aux professionnels du transport routier. De la même manière, ses syndicats locaux ou elle-même assurent un service d'aide juridique ou, via des sociétés de services, une aide matérielle aux entreprises de transport routier (COULOUARN,
2008) ${ }^{(10)}$. Il s'agit d'ailleurs, la plupart du temps, d'une présentation à plat de la réglementation existante.

Cependant, à lire les éditoriaux, comme les discours de clôture des congrès, les questions sociales, tout en étant présentes, ne forment plus qu'une part fort réduite du contenu. Cette discrétion des thèmes sociaux dans les préoccupations apparentes de l'organisation va aussi de pair avec un discours consensuel sur cette question ${ }^{(11)}$. En fin de compte, si l'OTRE communique peu et développe un langage consensuel en matière sociale, c'est parce qu'elle impute largement la responsabilité de la situation sociale dans le secteur non pas aux partenaires sociaux mais à l'incurie de l'État, considéré comme principal responsable de la crise du transport routier en France.

(10) Sur les services apportés à leurs membres par les organisations patronales, $c f$. COULOUARN, 2008. On sait que dans une optique économiste, ces avantages contribuent à accentuer le coût d'une non-participation à un mouvement social et, inversement, à diminuer le coût de cette participation : il s'agit «d'incitations sélectives» (Olson, 1978).

(11) Dans ses éditoriaux, l'organisation soutient parfois l'idée d'une communauté d'intérêts entre salariés du transport et les PME du secteur. 
Ce qui domine largement le discours de l'OTRE est bien une action revendicative d'ordre économique à l'encontre de l'État législateur (12). Mais alors que la participation au Comité national routier ou aux commissions consultatives de délivrance des attestations de capacité professionnelle pourrait être stratégique pour la défense des intérêts de la profession, au titre de la politique des transports, force est de constater que l'entrée dans ces institutions n'est jamais abordée par l'organisation, tant aux congrès annuels que dans ses publications.

Dans ses propos, l'OTRE présente l'État tout à la fois comme l'interlocuteur central et l'adversaire principal. Ce qu'elle lui reproche originellement, c'est l'absence de prise en compte, au niveau gouvernemental, de l'incidence de l'ouverture du marché européen sur la situation commerciale, économique et financière du «pavillon français ». Le cœur de l'action de l'OTRE consiste à obtenir de la part du gouvernement des modifications législatives et réglementaires sur les sujets les plus divers de l'économie des transports ${ }^{(13)}$. Les éditoriaux sont largement consacrés aux insuffisances dont font preuve les pouvoirs publics sur de multiples questions financières (fiscalité routière, péages autoroutiers, etc.), commerciales (cabotage, délais de paiement, etc.) ou techniques (caractéristiques des véhicules, interdictions de circulation, etc.). $\mathrm{Ce}$ sont également ces thèmes qui apparaissent régulièrement dans la correspondance qui se noue entre les services de l'État et l'organisation en des termes souvent extrêmement virulents ${ }^{(14)}$ tandis qu'elle engage ponctuellement des actions de terrain (envoi groupé de lettres de protestation, concentration de camions sur la voie publique, etc.) dont les modes opératoires l'éloignent, par exemple, de ceux que pourrait lui permettre son intégration au sein du Comité national routier.

(12) Si les salariés ont d'abord en face d'eux des employeurs, lesdits employeurs sont des chefs d'entreprise qui ont à faire à la fois à des entreprises concurrentes, des entreprises clientes et à une administration qui régule et encadre l'ensemble des facettes de leur activité.

(13) L'OTRE souhaiterait-elle des évolutions en matière de législation sociale, elle serait amenée à tenir compte du fait que celles-ci sont d'abord des évolutions réglementaires, avant d'être des évolutions de nature conventionnelle, commandées par une participation aux débats en commission mixte paritaire. Il est ici utile de rappeler qu'au XIXe siècle, la défense d'intérêts économiques communs, notamment face à l'État (questions douanières), a été au fondement des regroupements patronaux, même si les préoccupations sociales (paternalisme) n'ont pas été absentes.

(14) Par exemple, dans un style sarcastique : «Les transporteurs n'attendaient que ça, Bussereau leur a offert! La fameuse clause de répercussion gazole et son obligation pénale! Enfin, nous sommes sauvés!!! Désormais, grâce à la manne divino-ministérielle les clients qui ne voudront pas appliquer au transporteur la clause d'indexation gazole encourront une amende de $15000 €$ !!!! WAOUHHHH!! Je les vois déjà tremblant de peur!» (Newsletter, $\mathrm{n}^{\circ} 325$, semaine du 21 au 27 avril 2008).
Le champ d'action de l'OTRE apparaît donc en faible corrélation avec celui qui serait le sien si elle parvenait à obtenir une reconnaissance administrative de représentativité au niveau national. Pourtant, et c'est là que réside le paradoxe, elle a mené une quête constante et obstinée pour l'obtenir.

\section{Une apparente contradiction : la quête obstinée d'une reconnaissance de représentativité}

L'Organisation des transporteurs routiers européens apparaît début octobre 2000 (voir encadré 1). Dès le 31 octobre, une demande est officiellement reçue au ministère du Travail afin que soit reconnue la représentativité de l'organisation. Cette première demande ne va pas aboutir.

Au tournant des années 2002 et 2003, l'OTRE continue d'intervenir auprès des ministères du Travail et des Transports pour faire évoluer ce dossier, comme en témoignent des lettres d'élus ayant intercédé en faveur de l'organisation, mais aussi plusieurs tentatives de l'OTRE pour que ses représentants soient reçus officiellement par le ministre de tutelle ou par l'un de ses proches collaborateurs. Or la réouverture du dossier suppose une nouvelle demande. Celle-ci n'intervient officiellement que le 12 août 2003 et va également échouer. Le délai des six mois impartis pour l'enquête s'achève en février 2004 sans qu'une réponse ne soit donnée par l'Administration. L'organisation demande alors, en avril 2004, à ce que lui soient communiquées les raisons d'un silence valant rejet puis conteste cette décision administrative devant les tribunaux ${ }^{(15)}$.

Ces procédures contentieuses n'arrêtent pas pour autant les efforts de l'organisation pour faire avancer son dossier de reconnaissance de représentativité nationale. Dans le même temps, les éditoriaux des publications de l'OTRE et les discours des congrès

(15) Concernant la première demande de reconnaissance, il semble que l'alternance politique (élections présidentielles et législatives de mai et juin 2002) ait été un facteur d'enlisement obligeant l'organisation à en adresser une deuxième, le silence de l'Administration valant alors refus, tandis que sa demande d'explication sur un dossier ouvert sous l'égide d'un ancien gouvernement pouvait être tactiquement maladroite. Quant à la seconde demande, la raison officielle et principale du refus de l'Administration réside dans le déséquilibre territorial de l'implantation de l'OTRE, faiblement présente dans la moitié nord de la France. 
abordent très souvent cette question ${ }^{(16)}$. Le contentieux l'opposant à l'État sur la deuxième demande de reconnaissance de représentativité est tranché en avril 2007, à ses dépens. Si la période allant de l'été 2005 à mi-2007 apparaît moins active sur la question tout en marquant un certain durcissement dans les rapports entre l'OTRE et le ministère de tutelle, il semble qu'un rapprochement sur le sujet entre l'État et l'organisation va progressivement s'opérer, sous l'influence de différents facteurs ${ }^{(17)}$. C'est ainsi que le 26 octobre 2008, l'OTRE dépose auprès $\mathrm{du}$ ministère $\mathrm{du}$ Travail une troisième demande de reconnaissance de représentativité nationale, reconnaissance qui lui est finalement acquise par la décision du 31 août 2009(18).

Pourquoi une telle focalisation sur l'obtention d'un label d'apparence si technique? Parce qu'une reconnaissance administrative de représentativité au niveau national conduit effectivement à une reconnaissance générale de l'organisation - qui devient alors un partenaire officiel - et va en cela bien au-delà de ce qu'en disent les quelques textes juridiques sur le sujet(19). Elle a également une portée symbolique non négligeable car aux yeux de l'organisation, son obtention consacre la justesse des positions qu'elle défend en lui apposant ce qui pourrait s'apparenter à un label qualité( 20$)$.

(16) Par exemple, lors des premiers congrès, la présence ou l'absence d'un représentant officiel du ministère des Transports, comme signe de reconnaissance dont l'obtention de la représentativité officielle serait le parangon, est un thème récurrent des discours de clôture : «une fois de plus le ministre des Transports n'est pas parmi nous... comme à Carcassonne... et à Toulon. Pourtant l'OTRE représente par son nombre de transporteurs adhérents la $2^{\mathrm{e}}$ organisation professionnelle du transport de marchandises. Monsieur le ministre, pourquoi ne pas avoir envoyé quelqu'un vous représenter»? (Congrès 2003, discours de clôture, Jean-Pierre Morlin).

(17) Retour au ministère chargé des Transports de Dominique Bussereau (secrétariat d'État, juin 2007), rapprochement de différents syndicats régionaux de l'Unostra avec l'OTRE et crise concomitante de l'Unostra à l'automne 2009, départ de Jean-Pierre Morlin de la présidence de l'OTRE et remplacement à ce poste par Bernard Lataste lors du congrès de Biarritz en 2009.

(18) Cette reconnaissance de représentativité va faire l'objet d'un recours en annulation de la part de l'Union des fédérations de transport (UFT), de la FNTR et de l'Unostra devant le tribunal administratif de Bordeaux. Le 28 février 2012, le tribunal administratif annule l'acte du ministre du Travail pour erreur de droit, reprochant à l'État de ne pas avoir assez motivé sa décision. L'OTRE fait appel de cette décision. Parallèlement, l'Administration octroie de nouveau le label à l'OTRE le 7 septembre 2012.

(19) L'organisation obtient ainsi la possibilité d'agir plus efficacement sur l'évolution des règles et des pratiques régissant la profession (REYNAUD, 1997).

(20) Jean-Pierre Morlin, rappelant une rencontre, en novembre 2007, avec le secrétaire d'État aux Transports, indique : «en présence de tout le cabinet du secrétariat d'État [Dominique Bussereau] rendait un hommage appuyé au travail effectué par l'OTRE depuis sa création (...). Il concluait en assurant que la reconnaissance de notre organisation, désormais dans les tuyaux, n'était plus qu'une question de semaines » (Congrès de Nantes 2008).

\section{L'enjeu stratégique d'une reconnaissance de représentativité}

Afin d'obtenir la représentativité au niveau national, l'OTRE a concentré la plupart du temps son action au niveau politique et a agi comme si la décision de l'État revenait au seul ministre de tutelle sans tenir compte du rôle du ministère du Travail qui pourtant instruit les enquêtes de représentativité et prend des décisions officielles sur le sujet. Et, de fait, le rôle du ministère des Transports lors des enquêtes de représentativité est attesté par différents échanges ${ }^{(21)}$.

Pourquoi une telle différence de traitement? Il s'avère que ces enquêtes ont lieu à défaut d'un consensus entre partenaires sociaux sur la présence d'une organisation en commission mixte paritaire. En d'autres termes, la procédure officielle menée par le bureau des relations collectives du travail du ministère des Affaires sociales est précédée d'une procédure officieuse dans laquelle le ministère chargé des Transports joue un rôle moteur puisque la commission mixte paritaire des transports routiers se réunit au ministère des Transports et est présidée par un de ses fonctionnaires ${ }^{(22)}$. La même démarche est effectuée auprès des autres organisations syndicales dans le cadre, cette fois, de l'enquête officielle de représentativité, qui peut d'ailleurs apparaître comme une ultime tentative de parvenir à un consensus, tant l'opinion et l'avis des autres organisations sur une possible reconnaissance de représentativité du requérant semblent déterminés par le jeu des alliances et des oppositions, plutôt que par la mesure d'une représentativité effective. Nous sommes bien ici dans un processus de reconnaissance dans lequel la qualité des relations qu'entretiennent les différentes organisations joue un rôle tout aussi important que la représentativité de fait, qu'établirait l'enquête de l'Administration.

Quant à la décision finale, si elle revient officiellement au ministère du Travail (23), nous pouvons supposer qu'elle est prise en réalité par le ministre

(21) Lors de la première demande en reconnaissance de représentativité, une entrevue est organisée au ministère des Transports dès le 26 mars 2002. Toujours en 2002, un conseiller technique du secrétariat d'État aux Transports reçoit une délégation de l'OTRE, ce dont J.-P. Morlin le remercie par un courrier. L'entrevue aurait permis d'aborder «un point fondamental, notre reconnaissance (...) techniquement bouclée et dont vous trouverez ci-joint l'ensemble du dossier qui avait été déposé» auprès des services du ministère du Travail.

(22) Un courrier du bureau des relations collectives du travail du ministère du Travail adressé à l'Union des fédérations du transport (31 octobre 2008) indique qu'en l'absence «d'accord de l'ensemble des membres de la Commission mixte paritaire», il lui est demandé son avis à l'occasion de l'enquête de représentativité portant sur l'OTRE.

(23) En l'espèce, Xavier Darcos, ministre du Travail depuis juin 2009, décision communiquée par le directeur général du travail, Jean-Denis Combrexelle ( $«$ le ministre du travail... estime que votre organisation est représentative...»). 
chargé des Transports, qui ne se trouve pas forcément lié par l'enquête de représentativité. Il se trouve d'ailleurs que l'OTRE a été la première organisation patronale à demander que lui soit reconnu son caractère représentatif au niveau national pour le transport routier afin de siéger à la commission mixte paritaire, et pour laquelle une enquête de représentativité a été menée en bonne et due forme. Ni la FNTR, ni l'Unostra ni l'Union TLF n'ont fait l'objet d'une telle enquête. Seule la représentativité de l'Unostra a été reconnue officiellement, mais sans qu'il y ait eu enquête et par une décision émanant directement du ministre des Transports (24).

L'hypothèse est donc bien que, en présence d'un consensus entre les membres de la commission mixte paritaire sur l'accueil d'une nouvelle organisation, cette entrée équivaut à une reconnaissance de représentativité, le cas échéant formellement reconnue par une décision ministérielle. Quand bien même une enquête de représentativité a lieu, la décision ministérielle reste une décision administrative à la fois discrétionnaire et teintée d'opacité : une décision pour laquelle le ministre n'a pas un pouvoir lié, une opacité supplémentaire provenant du fait que la décision réelle, mais officieuse, appartient au ministre des Transports, alors que la décision officielle revient au ministre du Travail.

Le processus d'attribution du label de représentativité est donc éminemment politique et peut s'expliquer par ce qui est attendu des organisations qui l'obtiennent, qui va bien au-delà de ce que la législation fixe explicitement. La représentativité officielle au niveau national facilite la participation à des instances de représentation et de négociation, en plus des quelques instances nationales citées par les textes. Par exemple, alors qu'il n'est nullement exigé par la loi que les organisations professionnelles siégeant dans les Commissions régionales des sanctions administratives soient considérées comme représentatives, il est pourtant manifeste que l'OTRE va se heurter à de graves difficultés pour y siéger entre 2001 et 2009. Le cas de la Commission des sanctions administratives (CSA) d'Aquitaine est ici emblématique puisque le siège de l'OTRE se trouve à Bordeaux et, qu'en tout état de cause, une

(24) On peut faire l'hypothèse que la FNTR étant née du rapprochement de trois syndicats (dont la Fédération nationale des transporteurs routiers de France [FNTF], principal syndicat routier, apparue dès 1933) et l'Union TLF regroupant deux organisations fédérant les professions connexes du louage de véhicules (Chambre des loueurs et transporteurs industriels [CLTI]) et des commissionnaires de transport (Fédération française des organisateurs commissionnaires de transport [FFOCT]), il est apparu aux yeux de tous que ces formations étaient d'emblée représentatives. reconnaissance de représentativité au niveau régional aurait pu être acquise ${ }^{(25)}$.

Et il semble bien que les difficultés rencontrées par l'OTRE pour intégrer les Commissions des sanctions administratives se soient un peu partout présentées. Au niveau national, il est certain qu'aucune des instances officielles n'a admis l'OTRE durant les dix premières années de son existence, qu'il s'agisse notamment du Conseil national des transports ou du Comité des usagers du réseau routier national, pour lesquels aucune exigence de représentativité n'est pourtant inscrite dans les textes. Par ailleurs, les organismes de prévoyance ou du champ de la formation professionnelle (Carcept, OPCA ${ }^{(26)}$ Transport, etc.) n'ont pas non plus accueilli l'OTRE durant cette décennie dans leur conseil d'administration. De plus, les dirigeants de l'organisation ont même remarqué que plus l'instance de concertation avait un caractère officiel et se trouvait en relation directe avec le ministère de tutelle, moins l'OTRE pouvait avoir un accès ouvertement. On peut donc faire l'hypothèse que l'absence de représentativité officielle au niveau national a été un frein jusque dans les rapports non institutionnels qui s'instaurent souvent entre une organisation professionnelle et les services de l'État. En effet, compte tenu des effets symboliques liés à la reconnaissance de représentativité au niveau national et des enjeux réels qu'elle entraîne, il devient difficile à l'État de refuser la participation d'une organisation à des échanges informels dès lors qu'elle est reconnue représentative, que ce soit au niveau local ou national. À l'inverse, l'absence de reconnaissance de représentativité peut faciliter la mise à l'écart d'une organisation surtout lorsqu'elle rencontre l'hostilité de structures concurrentes, ce qui a pour conséquence d'empêcher tout dialogue, qu'il soit souhaité par l'État ou par d'autres organisations.

Notons que l'OTRE, finalement reconnue représentative au niveau national, a pu participer pleinement aux «États généraux du transport routier», initiés en décembre 2009 par le secrétariat d'État aux Transports. Or les discussions qui s'y sont déroulées sont allées bien au-delà d'une rénovation de la convention collective et ont rassemblé non seulement les partenaires sociaux membres de la commission mixte paritaire mais également des représentants de l'État et des représentants des chargeurs. Elles doivent donc être considérées non comme l'expression d'un organisme officiel de négociation, ouvert aux seuls mandataires des organisations professionnelles reconnues représentatives

(25) En principe, il est demandé aux organisations syndicales d'être représentatives (quand cette exigence existe) au niveau même où s'exerce leur représentation. De la sorte, l'absence d'une représentativité interprofessionnelle n'empêche nullement l'organisation d'exercer ses prérogatives au niveau d'une branche dès lors qu'elle est représentative dans celle-ci. (26) Organisme paritaire collecteur agréé. 
dans le champ du secteur du transport routier de marchandises, mais comme les fruits d'un forum informel auquel l'organisation a été officiellement invitée.

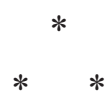

La reconnaissance sociale d'une organisation professionnelle ne passe pas nécessairement par sa représentativité effective : tout dépend de l'état des relations entre les partenaires. C'est pourquoi l'OTRE a pu conclure des alliances avec l'Union TLF et l'Unostra alors qu'elle a sans doute accentué dans le même temps son discours offensif envers l'État, précisément parce qu'elle s'est vue refuser, pendant dix ans, une pleine reconnaissance juridique de la part des pouvoirs publics, qui lui barrèrent l'accès à différents forums et lieux de discussion (Grossman, SaurugGer, 2006; Offerlé, 2009; COURTY, 1993).

Ce schéma des relations, tant institutionnelles qu'informelles, laisse de côté le rôle que peut jouer la reconnaissance officielle de représentativité au niveau national. Si en apparence la reconnaissance juridique de représentativité n'est qu'un élément parmi d'autres de la reconnaissance pleine et entière de l'organisation auprès des pouvoirs publics, cette reconnaissance en droit au niveau national peut néanmoins inverser radicalement l'ordre des choses en étant non seulement le sésame d'une reconnaissance pleine et entière de la part des pouvoirs publics - et non plus uniquement la reconnaissance informelle de l'organisation auprès du corps social - mais en devenant également la marque véritable de cette reconnaissance aux yeux mêmes de l'organisation (27). Obtenir une reconnaissance officielle de représentativité est manifestement aux yeux des adhérents et des dirigeants de l'OTRE la réparation d'une injustice qui leur a été causée (28). Issus pour la plupart de la "fédération historique», qui

(27) Dans le discours de l'OTRE, cette proximité sémantique apparaît régulièrement, de même qu'une confusion entre reconnaissance (sociale) et reconnaissance de représentativité, et par conséquent entre l'admission en commission mixte paritaire (relation entre partenaires sociaux), et la possibilité d'une collaboration avec l'État et ses partenaires qui va au delà des partenaires sociaux : "Quant à l'OTRE, il lui faut souhaiter que le processus enclenché par le secrétaire d'État aux transports, monsieur Bussereau, en la recevant officiellement au mois de novembre dernier, trouve son épilogue dans l'intégration rapide de notre organisation à la table des négociations paritaires. Ainsi entièrement habilitée à travailler avec ses interlocuteurs publics...» (J.-P. Morlin, Informations diverses, 31 décembre 2007, éditorial).

(28) Sur la nécessité d'une "expérience négative» pour la possible constitution d'une action revendicative, voir VoIROL, 2009. De façon approchante, sur les mobilisations identitaires («mouvements de statuts») des groupes sociaux ayant pu faire l'objet d'un ostracisme et cherchant à retrouver la considération qu'ils estiment mériter, voir NEVEU, 2011. fut et reste un interlocuteur reconnu et labellisé des pouvoirs publics, exclus de cette fédération, ils ont pu avoir le sentiment de ne retrouver leur place qu'à la condition d'être traités par les pouvoirs publics sur un strict plan d'égalité avec la FNTR.

Élément ultime et durable de reconnaissance, la représentativité est soumise à la capacité préalable des différents partenaires de s'admettre mutuellement, d'entrer dans le jeu d'une reconnaissance réciproque, nécessaire tant à l'action publique de l'État qu'à l'action conjointe des organisations envers les institutions publiques (LASCOUMES, LE GALÈs, 2007). Elle mène à un cercle fermé. En l'état actuel de la législation, l'obtention de la représentativité officielle d'une organisation patronale tend à être pérenne : aucune autre enquête, aucune élection périodique, ne viendra vérifier à l'avenir la réalité de cette représentativité, sauf à ce qu'il y ait une contestation devant les tribunaux.

La représentativité officielle justifie que le syndicat puisse représenter la profession au-delà de ses adhérents. En effet, la reconnaissance de représentativité peut être pensée comme un mécanisme permettant de dépasser les limites d'un double mandat : celui des adhérents de l'organisation envers ses dirigeants, mandatés pour les représenter en toute circonstance; celui des représentants du syndicat au sein d'une instance de concertation, mandatés expressément pour ce cas unique. La reconnaissance de représentativité autorise le syndicat à se considérer à l'image du corps social qu'il représente. Par-delà les limites propres à la technique du mandat, la pratique de la reconnaissance administrative de représentativité permettrait à l'organisation professionnelle de jouer pleinement un rôle d'intermédiation qui, en retour, justifie son existence auprès des membres d'un groupe professionnel, en l'occurrence les PME des entreprises du transport routier français (DUBAR, LUCAS, 1994; Dubar, Tripier, 1998). Cependant, dans le même temps, le caractère figé de cette représentation, à l'obtention aléatoire mais au maintien pérenne, pourrait aboutir à terme à de nouvelles tensions, les professionnels ne se reconnaissant plus nécessairement au travers des organisations finalement labellisées. L'émiettement de la représentation patronale pourrait être alors une conséquence, en forme de fuite en avant, aux recompositions du corps social de chaque secteur d'activité. 


\section{Bibliographie}

Berger P., Luckmann T. (2006), La construction sociale de la réalité, Paris, Armand Colin (1éd. 1966).

BunEl J. (1996), «Représentation patronale et représentativité des organisations patronales », Travail et emploi, $\mathrm{n}^{\circ} 70$, pp. 3-19.

Caillé A., Lazzeri C. (dir.) (2009), La reconnaissance aujourd'hui, Paris, CNRS éd.

Coulouarn T. (2008), Au nom des patrons, thèse de doctorat de science politique, Université Paris I.

COURTy G. (1993), Les routiers : contribution à une sociologie politique des groupes d'intérêt, thèse de doctorat de science politique, Université Paris X.

Courty G. (2006), Les groupes d'intérêt, Paris, La Découverte.

Dubar C., Lucas Y. (dir.) (1994), Genèse et dynamique des groupes professionnels, Lille, Presses universitaires de Lille.

Dubar C., Tripier P. (1998), Sociologie des professions, Paris, Armand Colin.

Dubar C. (2010), La socialisation, Paris, Armand Colin.

FLAMENT L. (2009), «La représentativité des organisations patronales », Droit social, avril, pp. 436-444.

FrAZER N. (2005), Qu'est-ce que la justice sociale? Reconnaissance et redistribution, Paris, La Découverte, Paris.

Grossman E., Saurugger S. (2006), Les groupes d'intérêt : action collective et stratégies de représentation, Paris, Armand Colin.
Heas F. (2011), «La représentativité des organisations salariées et patronales en droit français », Revue de droit du travail, $\mathrm{n}^{\circ} 2$, pp. 91-98.

Honneth A. (2000), La lutte pour la reconnaissance, Paris, éd. Du Cerf.

LAPIERRE J.-W. (1984), «L'identité collective, objet paradoxal : d'où nous vient-il?», Recherches sociologiques, t. 15, n 2-3, pp. 195-205.

Lascoumes P., Le Galès P. (2007), Sociologie de l'action publique, Paris, Armand Colin.

Le Crom J.-P., Maggi-Germain N. (dir.) (2011), La construction de la représentativité patronale, rapport pour la Dares, avril.

Neveu E. (2011), Sociologie des mouvements sociaux, Paris, La Découverte, (5 éd.).

Offerle M. (2009), Sociologie des organisations patronales, Paris, La Découverte.

Olson M. (1978), Logique de l'action collective, Paris, Presses universitaires de France, coll. «Sociologies».

Surdez M., Voegti M., Voutat B. (dir.) (2010), Identifier, $s$ 'identifier : à propos des identités politiques, Lausanne, éd. Antipodes.

Reynaud J.-D. (1997), Les règles du jeu : l'action collective et la régulation sociale, Paris, Armand Colin, coll. «Collection U».

Voirol O. (2009), «De l'expérience négative à l'agir politique : construction et clôture des causes collectives », in Guillaud Y., Widmer Y. (dir.), Le juste et l'injuste. Émotions, reconnaissance et action collective, Paris, L'Harmattan, pp. 11-130. 